Z Gerontol Geriat 2018 $51: 492$

https://doi.org/10.1007/s00391-018-1394-8

Online publiziert: 25. April 2018

(c) Springer Medizin Verlag GmbH, ein Teil von Springer Nature 2018

CrossMark

Roland Hardt ${ }^{1}$ Philipp Bahrmann ${ }^{2}$

'Universitätsmedizin der Johannes Gutenberg-Universität, Mainz, Deutschland

${ }^{2}$ Med. Klinik II, Asklepios Paulinen Klinik, Wiesbaden, Deutschland

\title{
Addendum zu: Chronische Herzinsuffizienz beim älteren Patienten
}

\section{Aktualisierte Nationale VersorgungsLeitlinie "Chronische Herzinsuffizienz" aus geriatrischer Perspektive}

\author{
Addendum zu: \\ Z Gerontol Geriat 2018 \\ https://doi.org/10.1007/s00391-018- \\ 1371-2
}

Der Übersichtsartikel zu der aktualisierten VersorgungsLeitlinie „Chronische Herzinsuffizienz" [1] aus geriatrischer Perspektive - bedarf aus Sicht der Autoren einer wichtigen Ergänzung: Im Abschn. „Medikamentöse Therapie“ wurde die Standardtherapie mit Angiotensin-Converting-Enzyme(ACE)Hemmern (alternativ ARB [AngiotensinRezeptor-Blocker]), $\beta$-Rezeptoren-Blockern und Mineralokortikoidrezeptorantagonisten, bei Flüssigkeitsretention ergänzt durch Diuretika, entsprechend der aktuellen Nationalen VersorgungsLeitlinie (NVL) zwar korrekt wiedergegeben. Es unterblieb jedoch der Hinweis aus der NVL, dass bei „Patienten, die trotz leitliniengerechter Therapie mit ACEHemmern, Betarezeptorenblockern und Mineralokortikoidrezeptorantagonisten symptomatisch sind, (...) ein Wechsel von ACE-Hemmern auf Sacubitril/ Valsartan empfohlen werden" sollte. Ein Einfluss des Alters auf den Effekt von Sacubitril/Valsartan fand sich in der dieser Empfehlung zugrunde liegenden Studie (PARADIGM-HF) nicht, sodass diese auch für geriatrische Patienten uneingeschränkt gültig ist. Die Autoren bedauern die fehlende Erwähnung dieser wichtigen therapeutischen $\mathrm{Al}$ - ternative und bitten, dieses Addendum bei der Therapie der Herzinsuffizienz mit eingeschränkter Ejektionsfraktion $\mathrm{zu}$ berücksichtigen. Keinesfalls sollte der Eindruck erweckt werden, dass ausgerechnet die Vertreter der Deutsche Gesellschaft für Geriatrie (DGG) bei der Überarbeitung der NVL die geriatrischen Patienten vom medizinischen Fortschritt ausschließen wollten.

\section{Korrespondenzadresse}

Prof. Dr. R. Hardt

Universitätsmedizin der Johannes GutenbergUniversität

Mainz, Deutschland

Roland.Hardt@unimedizin-mainz.de

Interessenkonflikt. P. Bahrmann und R. Hardt geben an, dass kein Interessenkonflikt besteht.

\section{Literatur}

1. Nationale VersorgungsLeitlinie Chronische Herzinsuffizienz, Langfassung, 2. Auflage 2017, Version 2,AWMF-Register-Nr.: nvl-006 (Pkt. 6.2.2.1 (S.61)

Die Online-Version des Originalartikels ist unter https://doi.org/10.1007/s00391-018-1371-2 zu finden. 